\section{Paracoccidioidomicosis con afectación mucocutánea. Reporte de un caso}

\author{
Paracoccidioidomycosis with \\ mucocutaneous involvement. A case report
}

\section{Resumen}

La Paracoccidioidomicosis es una micosis sistémica causada por el hongo dimórfico Paracoccidioides brasiliensis, con alta incidencia en Brasil y América Latina. La enfermedad se clasifica clínicamente en una forma juvenil - aguda y una forma adulta crónica. La lesión primaria se inicia en los pulmones a través de la inhalación de Paracoccidioides brasiliensis por el tracto respiratorio. Puede extenderse por vía hematógena y linfática a otros órganos, así como la piel y las mucosas. Las manifestaciones en piel y mucosas se caracterizan por la formación de lesiones granulomatosas, organizadas de acuerdo con el tipo de patrón de respuesta inmune. Las personas que trabajan en la agricultura y viven en zonas rurales se encuentran en alto riesgo de infección. El diagnóstico se basa en la identificación directa del hongo con el microscopio, el cultivo o la histología del espécimen clínico. Además, las técnicas de biología molecular como la reacción en cadena de la polimerasa (PCR) demuestran una alta sensibilidad, adecuada para el diagnóstico y el seguimiento del tratamiento. La elección del tratamiento depende del estado de la enfermedad y se realiza con derivados de azol, como la anfotericina B y las sulfonamidas. Se reporta el caso de un paciente varón, agricultor, sin antecedentes sistémicos contributorios, que inhaló Paracoccidioides brasiliensis. Presentó lesiones granulomatosas en la cavidad oral, se realizó los exámenes clínicos, imagenológicos e histopatológicos, llegando al diagnóstico de paracoccidioidomicosis con afectación mucocutánea. Fue tratado con éxito en el servicio de cirugía bucal y maxilofacial del Hospital Nacional Hipólito Unanue Lima-Perú.

Palabras clave: América del Sur; Enfermedades pulmonares fúngicas; Paracoccidioidomicosis.

\begin{abstract}
Paracoccidioidomycosis is a systemic mycosis caused by the dimorphic fungus Paracoccidioides brasiliensis, with a high incidence in Brazil and Latin America. The disease is clinically, classified in a juvenile - acute form and a chronic adult form. The primary lesion starts in the lungs through the inhalation of Paracoccidioides brasiliensis through the respiratory tract. It may spread hematogenously and lymphatically to other organs, as well as the skin and mucous membranes. Skin and mucosa manifestations are characterized by the formation of granulomatous lesions, organized according to the type of host"s pattern of immune response. People that works in agriculture and lives in rural areas are considered high risk of infection group. The diagnosis is based on direct identification of the fungus with the microscope, culture or histology sample of the clinical specimen. In addition, molecular biology techniques such as polymerase chain reaction (PCR) demonstrate high sensitivity, considered appropiate for diagnosis and follow-up treatment. The choice of treatment depends on the state of the disease and is medicated with azole derivatives such as amphotericin B and sulfonamides. It is reported the case of a male patient, a farmer, with no systemic contributor, who inhaled Paracoccidioides brasiliensis. He presented granulomatous lesions in oral cavity, clinical, imaging and histopathological examinations were carried out, to diagnose paracoccidioidomycosis with mucocutaneous affectation. He was successfully treated at the oral and maxillofacial surgery service of the National Hospital Hipólito Unanue Lima-Peru.
\end{abstract}

Keywords: Lung diseases, fungal; Paracoccidioidomycosis; South America.

\section{Caso Clínico}

Christian Farfán Gutiérrez 1,a, David Moreno Villalobos ${ }^{1, a, b}$, Gloria Arellano Porras ${ }^{1, a, b}$, Arturo Rodríguez Flores 1,a,b

${ }^{1}$ Hospital Nacional Hipólito Unanue. Lima, Perú. a Cirujano Dentista.

${ }^{\text {b } C i r u j a n o ~ B u c a l ~ y ~ M a x i l o f a c i a l ~}$

Correspondencia:

Christian Farfán Gutiérrez

Correo electrónico: christianjr_88_@hotmail.com

Jirón Tarma 119 - Dpto. 502, Lima 1, Perú.

\section{Coautores:}

David Moreno Villalobos

david.moreno.v@hotmail.com

Gloria Arellano Porras

vicky_arellano1603@hotmail.com

Arturo Rodríguez Flores

a.arturor@hotmail.com

Conflicto de intereses: Los autores declaran no tener conflictos de interés.

Fuente de financiamiento: Autofinanciado.

Fecha de recepción: 18/05/17

Fecha de aceptación: 05/09/17 


\section{Introducción}

La paracoccidioidomicosis es una micosis sistémica causada por el hongo dimórfico térmico Paracoccidioides brasiliensis ${ }^{1}$. Esta enfermedad es endémica en ciertos países del sur y de centroamérica con la más alta prevalencia observada en Brasil (aproximadamente $80 \%)^{2}$. En el Perú el reporte de esta enfermedad no es obligatorio, por lo cual, no se tiene datos exactos de su prevalencia, solo el reporte de casos ha permitido conocer la distribución geográfica en zonas tropicales de la ceja de selva y selva baja amazónica ${ }^{3,4}$. Esto constituye una escasa cobertura en el diagnóstico y por ende un retraso en el tratamiento, lo que impide mejorar el pronóstico de esta enfermedad. Las personas que trabajan en la agricultura y viven en zonas rurales se encuentran en un riesgo particularmente alto de la infección ${ }^{1}$. La paracoccidioidomicosis aparece clínicamente en dos formas: una forma juvenil - aguda o subaguda y una adulta crónica ${ }^{2}$. Más del $90 \%$ de los casos son formas crónicas que afectan principalmente a los hombres entre 30-60 años de edad y se caracterizan por una progresión lenta durante meses o incluso años. Esta forma puede presentarse como unifocal, si sólo los pulmones se ven afectados (aproximadamente $25 \%$ de los casos), o multifocal, con diseminación extrapulmonar a la mucosa oral o nasal, a la piel, a los ganglios linfáticos o las glándulas suprarrenales. Con menor frecuencia, puede afectar el sistema nervioso, los huesos, el sistema circulatorio y ocasionar lesiones genitales ${ }^{2}$.

El diagnóstico se basa en la identificación directa del hongo con el microscopio, el cultivo o la evaluación histopatológica del espécimen clínico después de realizar una biopsia ${ }^{2}$.

Se utilizan tinciones especiales con PAS o a la metenamina de plata de Grocott-Gomoni, con el objetivo de mejorar la observación del hongo ${ }^{3}$. Además, las técnicas de biología molecular como la reacción en cadena de la polimerasa (PCR) demuestran una alta sensibilidad adecuada para el diagnóstico y el seguimiento del tratamiento ${ }^{4,5}$. Las pruebas serológicas se utilizan para la evaluación de la respuesta al tratamiento y recurrencia de la enfermedad ${ }^{6}$. Las sulfonamidas, el ketoconazol, el itraconazol, el fluconazol y la anfotericina B se han utilizado con éxito en el tratamiento de la paracoccidioidomicosis ${ }^{7}$. El itraconazol es el tratamiento de elección, siendo eficaz en más de $95 \%$ de los casos ${ }^{7}$. El cotrimoxazol todavía se utiliza con frecuencia, especialmente en la enfermedad crónica progresiva y como el mantenimiento después de un curso de la anfotericina $\mathrm{B}$ en casos graves de esta micosis 7 . La duración del tratamiento dependerá de la respuesta clínica, micológica, radiológica y serológica de la enfermedad ${ }^{8}$.

Se presenta el caso clínico de un paciente adulto mayor que fue diagnosticado con paracoccidioidomicosis con afectación mucocutánea, tratado con éxito en el servicio de cirugía bucal y maxilofacial de Hospital Nacional Hipólito Unanue.

\section{Reporte del caso}

Paciente varón de 64 años, natural y procedente del centro poblado de Cotahuacho, Andahuaylas - Apurímac - Perú, agricultor, sin antecedentes de inmunosupresión o compromiso sistémico. Aproximadamente hace ocho meses nota un aumento de volumen facial izquierdo, con predominio de labio superior e inferior y la aparición de "bolitas" en boca atribuyéndolo a la ingesta de coca y aguardiente. Con el paso del tiempo nota que las lesiones en la boca van aumentando en cantidad y tamaño, llegando a impedirle la apertura bucal con normalidad y dificultando la ingesta de alimentos tanto sólidos como líquidos. Acude al hospital de su localidad, donde le prescriben analgésicos y por recomendación médica acude al servicio de cirugía bucal y maxilofacial del Hospital Nacional Hipólito Unanue para el diagnóstico y tratamiento de su afección.

$\mathrm{Al}$ examen clínico extraoral se evidencia asimetría facial por una macroqueilia en el hemilabio izquierdo, superior e inferior así como la falta de continuidad del surco comisural izquierdo. A la evaluación intraoral se evidencia lesiones granulomatosas múltiples, de consistencia firme, bordes irregulares, base amplia, color rojizo, sangrantes a la palpación. Ubicadas en la mucosa del carrillo bucal izquierdo, mucosa de labio inferior, que se extienden hasta la encía alveolar de la primera premolar superior izquierda (pieza dentaria 2.4) La apertura bucal es limitada por el dolor y el sangrado a nivel de la mucosa del carrillo bucal izquierdo (Figura 1).

La evaluación clínica y la historia del paciente llevan al diagnóstico presuntivo de una enfermedad micótica, por lo cual se plantea como plan para el diagnóstico definitivo una biopsia incisional y una radiografía de tórax para corroborar la afectación pulmonar que tienen algunas enfermedades micóticas.

Se realiza la biopsia incisional, enviándose la muestra a anatomía patológica. El resultado de la evaluación microscópica revela múltiples granulomas con células gigantes multinucleadas que se distriubuyen en todos los haces musculares y el corión, dentro de las cuales se encuentran estructuras de doble membrana. $\mathrm{Al}$ realizar la coloración de PAS se revela la presencia de estructuras ovoides de doble membrana con gemación perriférica, formando la imagen de "rueda de timón" u "orejas de mickey mouse". Estas características microscópicas son contributorias de paracoccidioidomicosis (Figura 2).

La radiografía de tórax evidencia un infiltrado reticulonodulillar difuso bilateral lo que confirma junto con el resultado de anatomía patológica el diagnóstico de paracoccidioidomicosis (Figura 2). Todo indica que el paciente inhaló la espora cuando realizaba su trabajo cotidiano de agricultor y que la enfermedad primero se instauró de manera focal en los pulmones y posteriormente por vía hematógena llego a la vía mucocutanea. Al realizar exámenes de laboratorio ninguno mostró alteración contributoria al cuadro. Se realiza una interconsulta al servicio de infectología con quienes se acuerda iniciar el tratamiento con itraconazol de $100 \mathrm{mg} \mathrm{c} / 24$ horas por seis 

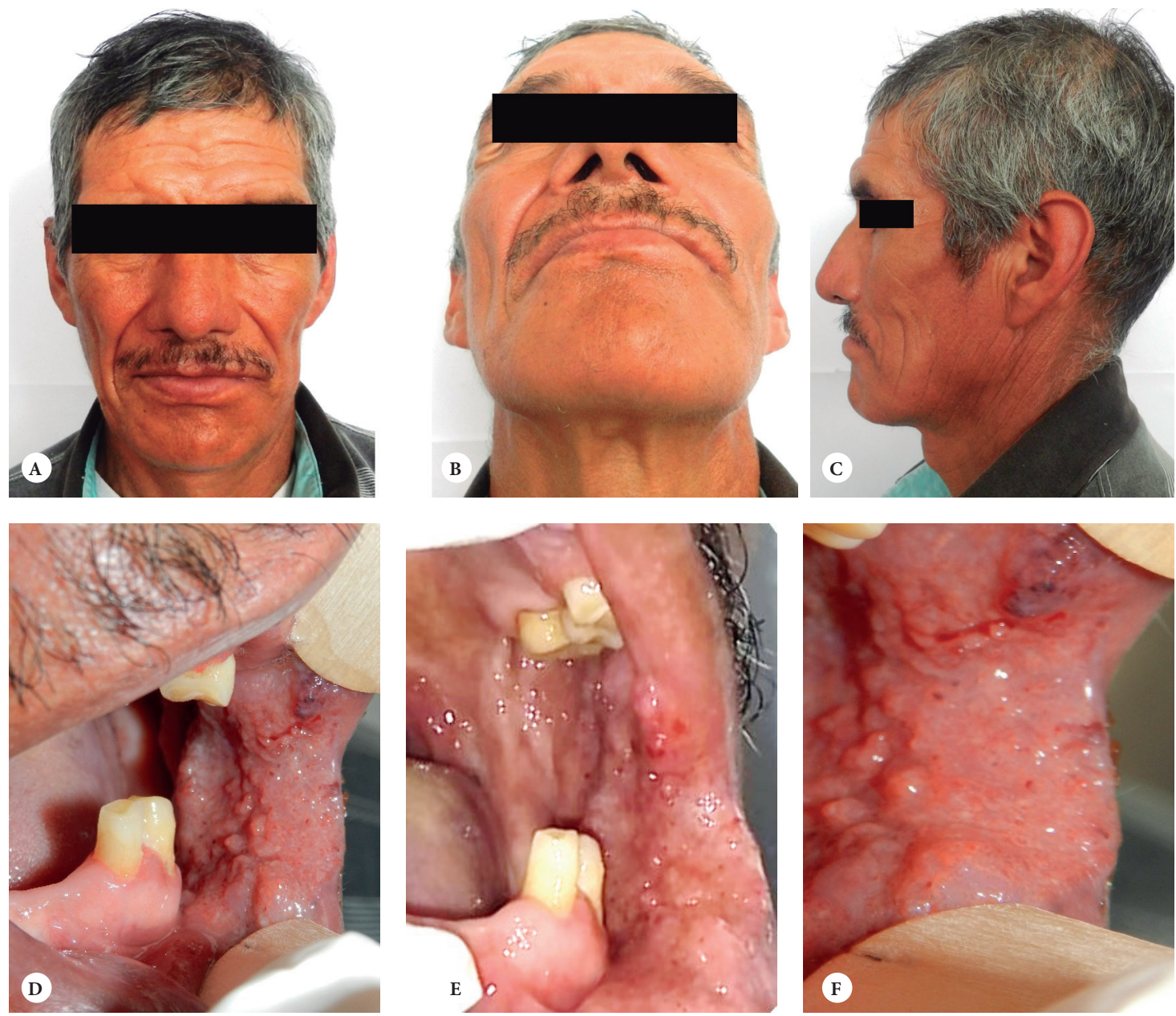

Figura 1. A), B) y C) Exámen extraoral que evidencia aumento de volumen en labio superior e inferior izquierdo y falta de continuidad de surco comisural izquierdo D), E) y F) Imagenes intraorales que muestras lesiones granulomatosas características, de consistencia firme, bordes irregulares, sangrantes, ubicadas en la mucosa del carrillo bucal izquierdo, mucosa de labio inferior y mucosa alveolar de la pieza dentaria 2.4

meses vía oral. Actualmente el paciente muestra una evolución favorable y acude periodicamente a sus controles (Figura 2).

\section{Discusión}

Paracoccidioides brasiliensis es endémica en varios países de América del Sur ${ }^{9,10}$. Santo la describe como infección micótica sistémica más importante en Brasil, donde se puede encontrar el mayor número de zonas endémicas de todo el mundo ${ }^{11}$. La tasa de incidencia anual es de 10-30 infecciones por millón de habitantes y la tasa media de mortalidad es de 1,4 por millón por año ${ }^{12}$. Es considerada un grave problema de salud pública en Brasil y en los países de América del Sur como Venezuela, Colombia, Argentina, Uruguay y Ecuador ${ }^{11}$. En el Perú la paracoccidioidomicosis no se reporta obligatoriamente, lo que hace que no se tenga la incidencia exacta ${ }^{13}$. Los estudios de reporte de casos contribuyen en el conocimiento de la distribución geográfica en zonas tropicales de la ceja de selva y selva baja amazónica en los departamentos de Ucayali (Pucallpa), Huánuco (Tingo María), Cerro de Pasco (Oxapampa), Junín (La Merced, Chanchamayo), Loreto (Iquitos, Maynas), San Martín
(Lamas), Cusco (Quillabamba), Ayacucho (Huanta), Huancavelica, Madre de Dios y Arequipa ${ }^{12,13}$. Los hombres son afectados con mayor prevalencia que las mujeres, en edades que oscilan de los 30 y los 60 ańos, en su gran mayoría son trabajadores de áreas rurales, agricultores y trabajadores de la construcción ${ }^{1,14}$.

El diagnóstico diferencial debe incluir enfermedades como la tuberculosis, la histoplasmosis y la coccidioidomicosis quienes coinciden en presentar manifestaciones en la cavidad oral y compromiso del sistema respiratorio ${ }^{8}$. El paciente de este caso fue probablemente infectado por inhalación cuando realizaba su trabajo de agricultor, en una zona con clima ideal para el crecimiento de Paracoccidioides brasiliensis. La primera manifestación en el pulmón, obviamente, se mantuvo sin diagnosticar y por lo tanto - de acuerdo con la literatura - parece que existió un período de latencia de varios años desde la infección hasta la primera presentación clínica ya con diseminación a nivel cutáneo. Las lesiones cutáneas se presentan en la boca (labios, encías, paladar), la faringe, la laringe y en la nariz. El diagnóstico consiste en la detección de elementos fúngicos sugestivos de Paracoccidioides brasiliensis a través de cultivo y biopsia donde histopatoló- 

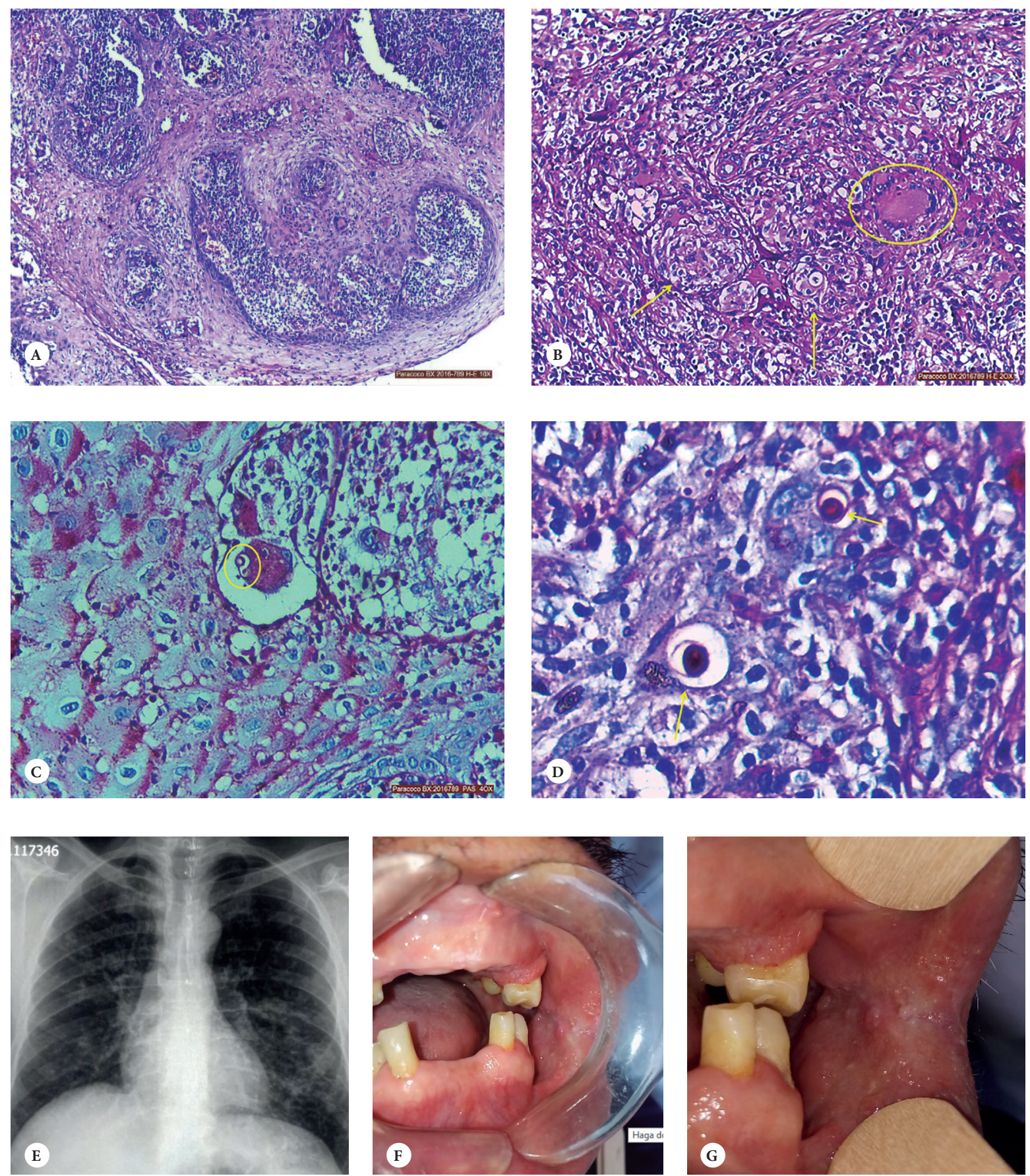

Figura 2. A) Epitelio con hiperplasia pseudoepiteliomatosa alrededor de granulomas, B) Granulomas epiteliodes (flechas) y Paracoccidioides brasiliensis, C) Paracoccidioides brasiliensis, con gemación periférica (círculo amarillo) "orejas de mickey mouse", D) Paracoccidioides brasiliensis dentro de macrófagos. Tinción PAS, E) Radiografía de tórax que muestra infiltrado reticulonodular difuso bilateral compatible con inoculación primaria de paracoccidioidomicosis, F) y G) Evaluación intraoral posterior a 2 meses de tratamiento con itraconazol, se evidencia una evolución favorable y la regresión de la lesiones granulomatosas.

gicamente se observará mayormente un epitelio plano estratificado, hiperplasia pseudoepiteliomatosa, muchas células gigantes donde los hongos muestran brotes hijos, conectados a sus células madre, en proceso de gemación, dando lugar a un aspecto clásico, ya bien descrito como de "rueda de timón" o de "orejas de Mickey Mouse" 15. También se recurre a tinciones especiales con PAS o a la metenamina de plata de Grocott, con el propósito de mejorar la observación del hongo ${ }^{1,16}$. La serología es útil no sólo para el diagnóstico, sino también para el segui- miento de la enfermedad, y permite evaluar, además, la respuesta al tratamiento ${ }^{1,2,17}$.

Es importante tener en cuenta para realizar el tratamiento el IX International Meeting on Paracoccidioidomycosis, realizado en San Pablo, Brasil, en octubre de 2005. Donde se llegó al consenso, basado en evidencia, que la droga de elección es el itraconazol. La anfotericina B sólo se prescribe para casos graves como tratamiento endovenoso inicial ( $1 \mathrm{mg} / \mathrm{kg} / \mathrm{día})^{4,5,10}$. El pronóstico va depender del estado de la enfermedad cuando se em- 
piece a tratar al paciente. El paciente de este reporte de caso fue tratado con itraconazol $100 \mathrm{mg} \mathrm{c} / 24 \mathrm{~h}$ por seis meses vía oral, mostrando una evolución favorable.

\section{Conclusiones}

Se concluye que un diagnóstico tardío de la enfermedad Paracoccidioidomicosis se relaciona con un deterioro progresivo de la calidad de vida del paciente, que puede terminar de manera fatal. Es de vital importancia conocer las manifestaciones clínicas y sistémicas de esta patología, ya que al asociarla a lugares endémicos nos lleva a un diagnóstico temprano y así instituir el tratamiento adecuado.

\section{Referencias bibliográficas}

1. Ameen M, Talhari C, Talhari S. Advances in paracoccidioidomycosis. Clin Exp Dermatol. 2013;35(6):576580 .

2. Brummer E, Castañeda A, Restrepo A. Paracoccidioidomycosis: an update. Clin Microbiol Rev. 1993;6(2):89117.

3. Bustinduy M, Guimerá F, Arévalos P, Castro C. Cutaneous primary Paracoccidioidomycosis. JEADV. 2009; 14:113-117.

4. Buitrago MJ, Merino P, Puente S, Gomez-Lopez A, Arribi A, Zancope-Oliveira RM. Utility of real-time PCR for the detection of Paracoccidioides brasiliensis DNA in the diagnosis of imported Paracoccidioidomycosis. Med Mycol. 2009;47(8):879-882.

5. Gomes GM, Cisalpino PS, Taborda CP, De Camargo ZP. PCR for diagnosis of paracoccidioidomycosis. J Clin Microbiol. 2000;38(9):3478-3480.

6. Wanke B, Aide MA. Chapter 6-paracoccidioidomycosis. J Bras Pneumol. 2009;35(12):1245-1249.

7. Colombo AL, Tobon A, Restrepo A, Queiroz-Telles F, Nucci M. Epidemiology of endemic systemic fungal infections in Latin America. Med Mycol. 2011;49(8):785798.
8. Ferreira M. Paracoccidioidomycosis. Paediatr Respir Rev. 2009; 10:161-165.

9. Pagliari C, Sotto MN. Dendritic cells and pattern of cytokines in Paracoccidioidomycosis skin lesions. Am J Dermatopathol. 2003;25:107e12.

10. Marques SA. Paracoccidioidomicose é esporotricose associada a Imunosupressão. Med Cutan Iber Lat Am. 2009;37:159e70.

11. Santo AH, Paracoccidioidomycosis-related mortality trend, state of São Paulo, Brazil: a study using multiple causes of death. Rev Panam Salud Publica. 2008; 23(5):313-24.

12. Subauste A, Castillo M, Torres R. Paracoccidioidomicosis pediátrica. Fol Dermatol Perú. 1997;8:30-8.

13. Burstein Z. Aspectos clínicos de la Blastomicosis sudamericana (Paracoccidioidomicosis) en el Perú. Rev Peru Med Exp Salud Pública. 2002;19(1):43-7.

14. Da Costa R, Miranda B, Camilo C. Juvenile paracoccidioimycosis in urban área: report of two cases. Infec Dis. 2010;14(1):77-80.

15. Venturini JV, Souza R, Moris DV. Altered disttibution of peripheal blood dendritic cell subsets in patients with pulmonary paracoccidioimycosis. Acta Trop. 2017; 173:185-190.

16. Buitrago MJ, Bernal-Martínez L, Castelli MV, Rodríguez-Tudela JL, Cuenca-Estrella M. Histoplasmosis and paracoccidioidomycosis in a non-endemic area: a review of cases and diagnosis. J Travel Med. 2011;18(1):26-33.

17. Shikanai-Yasuda MA, Telles Filho Q, Mendes RP, Colombo AL, Moretti ML. [Guidelines in paracoccidioidomycosis]. Rev Soc Bras Med Trop. 2006;39(3):297-310. 